Check for updates

Cite this: RSC Adv., 2017, 7, 31493

\title{
Biotransformation of dehydroepiandrosterone (DHEA) by environmental strains of filamentous fungi†
}

\author{
Ewa Kozłowska, (D) *a Monika Urbaniak, ${ }^{\mathrm{b}}$ Anna Kancelista, ${ }^{\mathrm{C}}$ Monika Dymarska, ${ }^{\mathrm{a}}$ \\ Edyta Kostrzewa-Sustow, ${ }^{a}$ Łukasz Stępień (DD ${ }^{\mathrm{b}}$ and Tomasz Janeczko (D) *a
}

\begin{abstract}
Microbial transformations of steroids are an important method of obtaining new steroid derivatives of potential pharmaceutical activity, which follows the principles of green chemistry. We studied the ability of selected filamentous fungus species to transform dehydroepiandrosterone (DHEA) and interesting DHEA derivatives were obtained. Twenty-five strains of filamentous fungi were isolated from soil and air in Wroclaw and they were used as biocatalysts. Strains of the genus Penicillium transformed the substrate into the products of Baeyer-Villiger lactonization in the D ring. Biotransformation of DHEA by Fusarium acuminatum KCh S1 strain led to a stereoselective hydroxylation at $7 \alpha$ (the product was

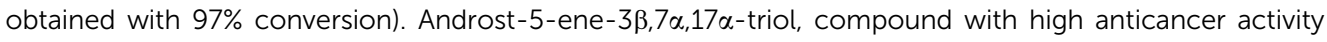
towards glioblastoma and lymphoma cell lines was obtained with $65 \%$ yield in the culture of Mucor hiemalis KCh W2 strain. In the culture of Aspergillus versicolor KCh TJ1 strain, DHEA was transformed into androst-1,4-diene-3,17-dione (ADD) with high isolated yield.
\end{abstract}

Received 24th April 2017

Accepted 13th June 2017

DOI: $10.1039 / \mathrm{c} 7 \mathrm{ra04608a}$

rsc.li/rsc-advances

\section{Introduction}

Steroid drugs, besides antibiotics, are the largest group of medicines that are used to treat and prevent various diseases. These compounds have anti-inflammatory, anti-allergic, immunomodulatory, anti-androgenic, anti-estrogenic, antitumor, antiviral, antibacterial, and hypotensive properties, as well as being widely known sex hormones. ${ }^{1-6}$

Dehydroepiandrosterone (DHEA) is a mammalian hormone, which is transformed in vivo into androgens and estrogens. ${ }^{7}$ The majority of DHEA found in blood is produced in the zona reticularis of the adrenal cortex. Very small amounts may be produced also in testicles, ovaries and brain cells. ${ }^{8}$ The majority of DHEA in mammalian blood is in the form of biologically inactive hydrophilic sulphate, which after hydrolysis by steroid sulphatase can be further transformed. ${ }^{9}$

DHEA's action is a result of combined influence on various receptors and enzymatic pathways. It is proven that DHEA and its metabolites can modulate the level of nitric oxide, reactive oxygen species and interleukin-2, as well as may interact with

${ }^{a}$ Department of Chemistry, Wrockaw University of Environmental and Life Sciences, Norwida 25, 50-375 Wroctaw, Poland. E-mail: e.a.kozlowska@gmail.com; janeczko13@interia.pl

${ }^{b}$ Department of Pathogen Genetics and Plant Resistance, Institute of Plant Genetics, Polish Academy of Sciences, Strzeszyńska 34, 60-479 Poznań, Poland

'Department of Biotechnology and Food Microbiology, Wroctaw University of Environmental and Life Sciences, Chetmońskiego 37, 51-630 Wroctaw, Poland

$\dagger$ Electronic supplementary information (ESI) available: ${ }^{1} \mathrm{H},{ }^{13} \mathrm{C}$ NMR, HSQC, COSY and MS data of all products. See DOI: 10.1039/c7ra04608a
Sigma-1 receptor, $\mathrm{G} \alpha_{1} 2$ and $\mathrm{G} \alpha_{1} 3$ receptors, peroxisome proliferator-activated receptor (PPAR)- $\alpha$, receptors for pregnane X (PXR), androstanol, estrogen $\beta$ (ER $\beta$ ), $\gamma$-aminobutyric acid type A $\left(\mathrm{GABA}_{\mathrm{A}}\right)$ and $N$-methyl-D-aspartate (NMDA).,10-12 This widespread mechanism of action can lead to multiple applications in the treatment of e.g.: elderly, menopause, osteoporosis, ovarian dysfunction, Crohn's disease, systemic lupus erythrematosus, asthma and Alzheimer's disease., ${ }^{\mathbf{8 1 3 , 1 4}}$ However, more side effects are recognised because of low DHEA specificity of action. Conversion of DHEA to androgens in female body leads to elevated testosterone level, which cause acne, increased facial, axillary and pubic hair growth. ${ }^{7,8,15}$ A solution could be the application of derivatives with greater specificity, higher activity than their precursors and no-sex hormone activity. $^{6}$

Due to widespread application of steroid compounds in medicine, there is a need for an efficient method for production of active pharmaceutical ingredients (APIs) and their key intermediates, and also for new derivatives with stronger biological activity. ${ }^{16}$ Biotransformations are good alternative to chemical synthesis in obtaining steroid compounds, allowing regio-and stereoselective introduction of substituents in nonactivated positions and the possibility to replace a several-step synthesis with a single microbial transformation. ${ }^{7}$ The most known example of using biotransformations in steroid synthesis was the improvement of a 39-step synthesis of cortisone from bile acids, which resulted in lowering production cost from $200 \$$ to $1 \$ .^{1} \mathrm{~A}$ very popular microbial transformation that found a large-scale application is the side-chain 
degradation in phytosterols derived from soybeans by bacteria of the genera Mycobacterium, Moraxella, and Rhodococcus, leading to progesterone or ADD (androst-1,4-diene-3,17dione). ${ }^{17}$ Steroid functionalization by hydroxylation leads to new derivatives with higher polarity and usually higher biological activity. ${ }^{\mathbf{1 8 , 1 9}}$ The most popular positions of hydroxylation of steroid skeleton are as follows: $6 \alpha, 6 \beta, 7 \alpha, 7 \beta, 9 \alpha, 11 \alpha, 11 \beta, 12 \alpha$, $12 \beta, 14 \alpha, 15 \alpha$ and $15 \beta$. Other carbons can be also hydroxylated. Fungi can also provide dehydrogenated and lactonised derivatives. ${ }^{1,17,20-22}$ Biotransformations of DHEA may become a lowcost and quick method to receive biologically active DHEA derivatives. $^{23,24}$

\section{Experimental}

\section{Materials}

The substrate - dehydroepiandrosterone (DHEA) was purchased from Sigma-Aldrich. The microorganisms used were: Penicillium chrysogenum KCh S4, Fusarium acuminatum KCh S1, Mucor hiemalis KCh W2, Penicillium commune KCh W7 and Aspergillus versicolor KCh TJ1. All strains were obtained from the Department of Chemistry of the Wrocław University of Environmental and Life Sciences and were isolated using the method described below.

\section{Isolation of strains}

Samples of soil from two landfill sites in Wrocław: Nadodrze district (S) and Słodowa Island, Old Town (W) were placed in sterile tubes. Under aseptic conditions $1 \mathrm{~g}$ of the soil was suspended in $10 \mathrm{~cm}^{3}$ of sterile water and shaken. Then $1 \mathrm{~cm}^{3}$ of the supernatant was taken out and serial decimal dilution was made. The inoculum from each of the dilutions was transferred into Sabouraud solid growth medium (3\% glucose, 1\% aminobac, $0.8 \%$ agar). From the (S) soil sample five pure cultures were isolated, whereas from the (W) soil sample seven strains were isolated. The strains that were the most effective in the screening tests were identified using molecular biology techniques and used in the reactions on a preparative scale. The results presented in this paper concern only the identified strains. A sample of air from the autoclave room in the Department of Chemistry (TJ) was used to inoculate agar plates using Koch sedimentation method. From the grown colonies 4 pure strains were obtained, from which the most effective one was the strain molecularly identified as Aspergillus versicolor KCh TJ1. The strain was maintained on Sabouraud 4\% agar slopes and freshly subcultured before use in the transformation experiments.

\section{DNA extraction and molecular identification of fungal strains}

Genomic DNA was extracted using a modified CTAB (cetyl hexadecyltrimethylammonium bromide) protocol, by the method described earlier. ${ }^{25}$ The DNA extracts were stored at $-20{ }^{\circ} \mathrm{C}$ until used. Species identification was done on the basis of the sequence of the Internal Transcribed Spacers of the ribosomal DNA region (ITS1-ITS2) (Table 1). PCR was done in 20 $\mu \mathrm{L}$ volumes using C-1000 thermal cyclers (Bio-Rad, Hercules, CA, USA). Each reaction tube contained $0.5 \mu \mathrm{L}$ of DreamTaq
Table 1 Sequences of primers used for molecular identification of fungal strains

\begin{tabular}{llll}
\hline \multirow{2}{*}{ Marker } & $5^{\prime}>3^{\prime}$ sequence & $\begin{array}{l}\text { Amplicon } \\
\text { size }(\mathrm{bp})\end{array}$ & Reference \\
\hline ITS4 & TCCTCCGCTTATTGATATGC & 600 & 26 \\
ITS5 & GGAAGTAAAAGTCGTAACAAGG & & \\
\hline
\end{tabular}

Green DNA polymerase (Thermo Scientific, Espoo, Finland), 2.5 $\mu \mathrm{L}$ of $10 \times$ DreamTaq Green Buffer, 12.5 pmol of forward/ reverse primers, $2.5 \mathrm{mM}$ of each dNTP, and about $20 \mathrm{ng}$ of fungal DNA. PCR conditions were as follows: $5 \mathrm{~min}$ at $94{ }^{\circ} \mathrm{C} ; 35$ cycles of $45 \mathrm{~s}$ at $94{ }^{\circ} \mathrm{C}, 45 \mathrm{~s}$ at $56{ }^{\circ} \mathrm{C}$, and $1 \mathrm{~min}$ at $72{ }^{\circ} \mathrm{C}$; and $5 \mathrm{~min}$ at $72{ }^{\circ} \mathrm{C}$.

Amplicons were electrophoresed in $1.5 \%$ agarose gel (Invitrogen) with GelGreen Nucleic Acid Stain (Biotium, Inc.).

For sequence analysis, PCR-amplified DNA fragments were purified with exonuclease I (Thermo Scientific) and shrimp alkaline phosphatase (Thermo Scientific) using the following programme: $30 \mathrm{~min}$ at $37{ }^{\circ} \mathrm{C}$ and $15 \mathrm{~min}$ at $80^{\circ} \mathrm{C}$. DNA fragments were labeled using forward primer and the BigDye Terminator 3.1 kit (Applied Biosystems, Foster City, CA, USA), according to producer's recommendations and precipitated with $96 \%$ ethanol. Sequence reading was performed using Applied Biosystems equipment. Sequences were analysed using BLASTn algorithm.

\section{Screening procedure}

Erlenmeyer flasks $(300 \mathrm{~mL})$, each containing $100 \mathrm{~mL}$ of the cultivation medium consisting of $3 \mathrm{~g}$ glucose and $1 \mathrm{~g}$ aminobac dissolved in water, were inoculated with a suspension of microorganisms and then incubated for 3 days at $25{ }^{\circ} \mathrm{C}$ on a rotary shaker. After full growth of the culture $10 \mathrm{mg}$ of a substrate dissolved in $1 \mathrm{~mL}$ of THF was added. After 1, 3 and 7 days of incubation under the above conditions, portions of 10 $\mathrm{mL}$ of the transformation mixture were extracted with $\mathrm{CHCl}_{3}(3$ $\times 10 \mathrm{~mL}$ ). The extracts were dried over $\mathrm{MgSO}_{4}$, concentrated in vacuo and analyzed by GC and TLC. All experiments were repeated three times.

\section{Preparative biotransformation}

The same transformations were performed on the preparative scale in $2000 \mathrm{~mL}$ flasks, each containing $500 \mathrm{~mL}$ of the cultivation medium. The cultures were incubated under the same conditions and then $100 \mathrm{mg}$ of DHEA dissolved in $2 \mathrm{~mL}$ of THF was added to the grown cultures. After complete transformation of the substrate the mixtures were extracted with $\mathrm{CHCl}_{3}(3 \times$ $300 \mathrm{~mL})$, dried $\left(\mathrm{MgSO}_{4}\right)$ and concentrated in vacuo. The transformation products were separated by preparative TLC and analyzed (TLC, GC).

\section{Analytical methods}

The course of biotransformation was controlled by means of TLC. Composition of product mixtures was established by GC. 
Products were purified using preparative TLC (silica gel GF, 20 $\times 20 \mathrm{~cm}, 500 \mu \mathrm{m}$, Analtech) and hexane/acetone mixture $(2: 1$, $\mathrm{v} / \mathrm{v}$ ) as eluent. After elution products were detected under UV light $(365 \mathrm{~nm})$, then scraped from the plate and eluted with acetone to give fraction. Analytical TLC was performed on silica gel G plates (Merck). Compounds were detected by spraying the plates with $\mathrm{H}_{2} \mathrm{SO}_{4} / \mathrm{CH}_{3} \mathrm{OH}$ mixture $(1: 1, \mathrm{v} / \mathrm{v})$ and visualized under UV (254 nm). GC analysis was performed using a HewlettPackard 5890A (Series II) GC instrument fitted with a flame ionization detector (FID). The DB-5MS (crosslinked phenyl methyl siloxane) capillary column $(30 \mathrm{~m} \times 0.32 \mathrm{~mm} \times 0.25 \mu \mathrm{m})$ was used to determine the composition of product mixtures. The following temperature programme was used: $220{ }^{\circ} \mathrm{C}(1$ $\min ) / 4{ }^{\circ} \mathrm{C} \min ^{-1} / 260{ }^{\circ} \mathrm{C}(1 \mathrm{~min}) / 30{ }^{\circ} \mathrm{C} \mathrm{min}^{-1} / 300{ }^{\circ} \mathrm{C}(5 \mathrm{~min})$. For GC-MS analysis, GCMS-QP2010 (Shimadzu) was used with a ZB5 (crosslinked phenyl methyl siloxane) capillary column $(30 \mathrm{~m}$ $\times 0.25 \mathrm{~mm} \times 0.25 \mu \mathrm{m})$. The following temperature programme was used: $180{ }^{\circ} \mathrm{C}(3 \mathrm{~min}) / 10{ }^{\circ} \mathrm{C} \mathrm{min}^{-1} / 300{ }^{\circ} \mathrm{C}(5 \mathrm{~min})$. The NMR spectra were recorded on DRX $500 \mathrm{MHz}$ Bruker spectrometer and measured in $\mathrm{CDCl}_{3}$.

\section{Results and discussion}

\section{Spectral data of isolated metabolites}

Product structures were determined by means of elemental analysis, ${ }^{1} \mathrm{H}$ NMR, ${ }^{13} \mathrm{C}$ NMR and correlation spectroscopy. ${ }^{13} \mathrm{C}$ NMR spectra of all the products obtained are summarized in Table 2 .

As a result of a 3 day transformation of DHEA (100 mg) with the strain Aspergillus versicolor KCh TJ1 androst-4-ene-3,17dione $(\mathrm{AD})(65.0 \mathrm{mg}, 65 \%)$ was isolated. After seven days of diene-3,17-dione (6ßOH-ADD) $(3.9 \mathrm{mg}, 4 \%)$ and $6 \beta$ hydroxyandrost-4-ene-3,17-dione (6ßOH-AD) $(2.3 \mathrm{mg}, 2 \%)$ were isolated. After a 3 day transformation with the strain Penicillium commune KCh W7 3 $\beta$-hydroxy-17a-oxa-D-homo-androst-5-ene17-one (3ßOH-lactone) (8.1 $\mathrm{mg}, 8 \%)$ and 17a-oxa-D-homoandrost-4-ene-17-one (testololactone) $(40.8 \mathrm{mg}, 41 \%)$ were isolated from the post-cultivation medium. Whereas, a 3 day transformation with the strain Penicillium chrysogenum KCh S4 led to isolation of androst-4-ene-3,17-dione (AD) (15.9 mg, 16\%) and 17a-oxa-D-homo-androst-4-ene-17-one (testololactone) (34.2 mg, 34\%).

A 3 day transformation of DHEA in the culture of Mucor hiemalis KCh W2 allowed for the isolation of androst-5-ene$3 \beta, 7 \alpha, 17 \alpha$-triol $\quad(3 \beta, 7 \alpha, 17 \alpha$-triol $) \quad(37.3 \mathrm{mg}, 38 \%), \quad 3 \beta, 7 \alpha-$ dihydroxyandrost-5-ene-17-one (7 $\alpha \mathrm{OH}-\mathrm{DHEA})$ (25.3 $\mathrm{mg}, 25 \%$ ) and $3 \beta$-hydroxyandrost-5-ene-7,17-dione (7Oxo-DHEA) $(2.2 \mathrm{mg}$, $2 \%$ ). Whereas, 7 days of transformation with the strain Fusarium acuminatum $\mathrm{KCh} \mathrm{S} 1$ gave $3 \beta, 7 \alpha$-dihydroxyandrost-5-ene17-one ( $7 \alpha \mathrm{OH}$-DHEA) (71.8 mg, 72\%).

Androst-4-ene-3,17-dione (AD). ${ }^{1} \mathrm{H}$ NMR (600 MHz) (ppm) $\left(\mathrm{CDCl}_{3}\right) \delta: 0.92$ (s, 3H, 18-H); $0.99(1 \mathrm{H}, \mathrm{ddd}, J=12.4,10.8$, $4.3 \mathrm{~Hz}, 9-\mathrm{H})$; 1.12 (qd, $1 \mathrm{H}, J=14.1,4.3 \mathrm{~Hz}, 7-\mathrm{H} \alpha)$; 1.21 (s, 3H, 19$\mathrm{H})$; $1.24-1.31(\mathrm{~m}, 2 \mathrm{H}, 12-\mathrm{H} \alpha, 14-\mathrm{H}) ; 1.46$ (qd, $1 \mathrm{H}, J=13.3$, $4.3 \mathrm{~Hz}, 11-\mathrm{H} \beta) ; 1.57$ (tt, $1 \mathrm{H}, J=12.4,9.3 \mathrm{~Hz}, 15-\mathrm{H} \beta) ; 1.67-1.73$ $(\mathrm{m}, 2 \mathrm{H}, 1-\mathrm{H} \alpha, 11-\mathrm{H} \alpha) ; 1.87$ (ddd, $1 \mathrm{H}, J=13.3,4.3,2.7 \mathrm{~Hz}, 12-$ $\mathrm{H} \beta$ ); 1.95-1.99 (m, 2H, 7-H $\beta, 15-\mathrm{H} \alpha$ ); 2.03 (ddd, $1 \mathrm{H}, J=13.3,4.8$, $2.7 \mathrm{~Hz}, 1-\mathrm{H} \beta) ; 2.56(\mathrm{dt}, 1 \mathrm{H}, J=18.8,9.1 \mathrm{~Hz}, 16-\mathrm{H} \alpha) ; 2.27-2.36$ $(\mathrm{m}, 3 \mathrm{H}, 2-\mathrm{H} \alpha, 2-\mathrm{H} \beta, 6-\mathrm{H} \alpha, 6-\mathrm{H} \beta) ; 2.46(\mathrm{dd}, 1 \mathrm{H}, J=19.3,8.9 \mathrm{~Hz}$, 16-H $\beta$ ); 5.75 (br s, $1 \mathrm{H}, 4-\mathrm{H}$ ).

Androst-1,4-diene-3,17-dione (ADD). ${ }^{1} \mathrm{H}$ NMR (600 MHz) (ppm) $\left(\mathrm{CDCl}_{3}\right) \delta: 0.93(\mathrm{~s}, 3 \mathrm{H}, 18-\mathrm{H}) ; 1.06-1.16(\mathrm{~m}, 2 \mathrm{H}, 7-\mathrm{H} \alpha, 9-$ $\mathrm{H}) ; 1.25$ (s, 3H, 19-H); 1.23-1.29 (m, 2H, 12-H $\alpha, 14-\mathrm{H}) ; 1.58(\mathrm{tt}$, $1 \mathrm{H}, J=12.4,9.2 \mathrm{~Hz}, 15-\mathrm{H} \beta$ ); 1.68 (qd, $1 \mathrm{H}, J=13.3,4.3 \mathrm{~Hz}, 11-$ $\mathrm{H} \beta) ; 1.80(\mathrm{td}, 1 \mathrm{H}, J=11.1,3.6 \mathrm{~Hz}, 8-\mathrm{H}) ; 1.82-1.88(\mathrm{~m}, 2 \mathrm{H}, 11-\mathrm{H} \alpha$, $12-\mathrm{H} \beta$ ); 1.95 (ddd, $1 \mathrm{H}, J=12.4,8.5,6.2 \mathrm{~Hz}, 15-\mathrm{H} \alpha$ ); 2.04-2.12

Table $2{ }^{13} \mathrm{C}$ NMR chemical shifts of products in $\mathrm{CDCl}_{3}$

\begin{tabular}{|c|c|c|c|c|c|c|c|c|c|}
\hline \multirow{2}{*}{$\begin{array}{l}\text { Atom } \\
\text { number }\end{array}$} & \multicolumn{9}{|c|}{ Products } \\
\hline & $\mathrm{AD}$ & $\mathrm{ADD}$ & $6 \beta \mathrm{OH}-\mathrm{AD}$ & $6 \beta O H-A D D$ & Testololactone & $7 \alpha \mathrm{OH}-\mathrm{DHEA}$ & $3 \beta, 7 \alpha, 17 \alpha-$ Triol & $3 \beta \mathrm{OH}$-lactone & 7OxO-DHEA \\
\hline 1 & 35.05 & 155.43 & 37.34 & 156.86 & 35.46 & 37.07 & 37.22 & 37.04 & 36.60 \\
\hline 2 & 33.81 & 127.82 & 34.28 & 127.03 & 33.84 & 31.39 & 31.10 & 31.59 & 31.25 \\
\hline 3 & 199.21 & 186.33 & 200.40 & 186.65 & 199.40 & 71.25 & 71.41 & 71.61 & 70.44 \\
\hline 4 & 124.04 & 124.24 & 126.58 & 126.17 & 124.07 & 42.06 & 42.19 & 42.01 & 41.99 \\
\hline 5 & 170.26 & 168.44 & 168.03 & 165.52 & 169.63 & 146.64 & 146.28 & 140.79 & 166.27 \\
\hline 6 & 32.46 & 32.65 & 72.83 & 73.70 & 32.35 & 123.67 & 123.99 & 120.70 & 126.06 \\
\hline 7 & 30.66 & 32.41 & 37.34 & 38.86 & 30.37 & 64.37 & 65.63 & 31.20 & 201.20 \\
\hline 8 & 35.58 & 35.22 & 29.52 & 29.93 & 37.92 & 37.32 & 37.98 & 34.58 & 44.47 \\
\hline 9 & 53.70 & 52.40 & 53.76 & 51.96 & 52.45 & 42.72 & 42.49 & 49.11 & 50.22 \\
\hline 10 & 38.56 & 43.54 & 38.17 & 43.62 & 38.46 & 37.63 & 37.60 & 36.92 & 38.54 \\
\hline 11 & 20.23 & 22.20 & 20.37 & 22.04 & 21.82 & 20.19 & 20.43 & 22.09 & 20.72 \\
\hline 12 & 31.19 & 31.30 & 31.37 & 31.34 & 38.93 & 31.18 & 31.51 & 39.04 & 30.85 \\
\hline 13 & 47.41 & 47.79 & 47.74 & 47.87 & 82.87 & 47.23 & 45.08 & 83.30 & 48.00 \\
\hline 14 & 50.71 & 50.53 & 51.00 & 50.63 & 45.62 & 45.05 & 42.13 & 46.85 & 45.88 \\
\hline 15 & 21.67 & 22.02 & 21.81 & 21.96 & 19.82 & 22.02 & 24.89 & 20.03 & 24.31 \\
\hline 16 & 35.67 & 35.74 & 35.88 & 35.80 & 28.53 & 35.91 & 32.75 & 28.95 & 35.77 \\
\hline 17 & 220.35 & 220.03 & 220.69 & 220.15 & 171.44 & 221.30 & 80.00 & 171.64 & 220.52 \\
\hline 18 & 13.62 & 13.92 & 13.88 & 14.01 & 17.40 & 13.39 & 16.76 & 20.20 & 13.89 \\
\hline 19 & 17.29 & 18.83 & 19.65 & 20.59 & 20.11 & 18.38 & 18.43 & 19.42 & 17.57 \\
\hline
\end{tabular}


(m, 2H, 7-H $\beta, 16-\mathrm{H} \alpha) ; 2.41$ (ddd, $1 \mathrm{H}, J=13.2,3.7,3.0 \mathrm{~Hz}, 6-\mathrm{H} \alpha)$; 2.46 (dd, $1 \mathrm{H}, J=19.4,9.0 \mathrm{~Hz}, 16-\mathrm{H} \beta) ; 2.50$ (dt, $1 \mathrm{H}, J=13.5$, $4.9 \mathrm{~Hz}, 6-\mathrm{H} \beta$ ); 6.08 (br s, $1 \mathrm{H}, 4-\mathrm{H}) ; 6.23$ (dd, $1 \mathrm{H}, J=10.2,1.2 \mathrm{~Hz}$, 2-H); 7.04 (d, 1H, $J=10.2 \mathrm{~Hz}, 1-\mathrm{H})$.

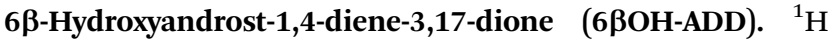
NMR (600 MHz) (ppm) $\left(\mathrm{CDCl}_{3}\right) \delta: 0.97$ (s, 3H, 18-H); 1.13 (d, 1H, $J=11.9,3.9 \mathrm{~Hz}, 9-\mathrm{H})$; 1.26-1.36 (m, 3H, 7-H $\alpha, 12-\mathrm{H} \alpha, 14-\mathrm{H}) ; 1.46$ (s, 3H, 19-H); 1.66 (dd, $1 \mathrm{H}, J=12.7,9.4 \mathrm{~Hz}, 15-\mathrm{H} \beta) ; 1.77$ (qd, $1 \mathrm{H}$, $J=13.4,4.2 \mathrm{~Hz}, 11-\mathrm{H} \beta)$; 1.84-1.92 (m, 2H, 11-H $\alpha, 12-\mathrm{H} \beta)$; $1.94-$ $2.01(\mathrm{~m}, 1 \mathrm{H}, 15-\mathrm{H} \alpha) ; 2.11(\mathrm{dt}, 1 \mathrm{H}, J=19.3,9.6 \mathrm{~Hz}, 16-\mathrm{H} \alpha) ; 2.19$ (d, $1 \mathrm{H}, J=14.0,3.1 \mathrm{~Hz}, 7-\mathrm{H} \beta$ ); 2.27 (ddd, $1 \mathrm{H}, J=14.4,11.1$, $3.7 \mathrm{~Hz}, 8-\mathrm{H})$; 2.49 (dd, $1 \mathrm{H}, J=19.4,8.9 \mathrm{~Hz}, 16-\mathrm{H} \beta) ; 4.58$ (br t, $1 \mathrm{H}$, $J=2.8 \mathrm{~Hz}, 6-\mathrm{H} \alpha) ; 6.17(\mathrm{~d}, 1 \mathrm{H}, J=1.5 \mathrm{~Hz}, 4-\mathrm{H}) ; 6.22(\mathrm{dd}, 1 \mathrm{H}, J=$ 10.2, 1.6 Hz, 2-H); 7.04 (d, 1H, $J=10.1 \mathrm{~Hz}, 1-\mathrm{H})$.

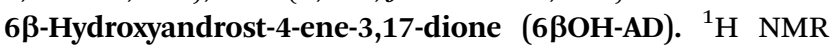
$(600 \mathrm{MHz})(\mathrm{ppm})\left(\mathrm{CDCl}_{3}\right) \delta: 0.93(\mathrm{~s}, 3 \mathrm{H}, 18-\mathrm{H}) ; 0.96(1 \mathrm{H}, \mathrm{ddd}, J=$ 12.5, 10.9, 4.2 Hz, 9-H); 1.25-1.34 (m, 3H, 7-H $\alpha, 12-\mathrm{H} \alpha, 14-\mathrm{H})$; 1.39 (s, 3H, 19-H); 1.50 (qd, $1 \mathrm{H}, J=13.1,4.2 \mathrm{~Hz}, 11-\mathrm{H} \beta) ; 1.61(\mathrm{tt}$, $1 \mathrm{H}, J=12.6,9.1 \mathrm{~Hz}, 15-\mathrm{H} \beta)$; 1.67 (ddd, $1 \mathrm{H}, J=13.7,7.0,3.9 \mathrm{~Hz}$, $11-\mathrm{H} \alpha$ ); 1.71 (ddd, $1 \mathrm{H}, 1 \mathrm{H}, J=14.8,13.2,4.0 \mathrm{~Hz}, 1-\mathrm{H} \alpha$ ); 1.86 (ddd, $1 \mathrm{H}, J=13.2,4.1,2.9 \mathrm{~Hz}, 12-\mathrm{H} \beta$ ); 1.98 (ddd, $1 \mathrm{H}, J=12.5$, 8.2, $5.9 \mathrm{~Hz}, 15-\mathrm{H} \alpha$ ); 2.03 (ddd, $1 \mathrm{H}, J=13.2,4.9,2.7 \mathrm{~Hz}, 1-\mathrm{H} \beta$ ); $2.12(\mathrm{dt}, 1 \mathrm{H}, J=19.5,9.2 \mathrm{~Hz}, 16-\mathrm{H} \alpha) ; 2.12$ (dt, $1 \mathrm{H}, J=10.9$, $3.2 \mathrm{~Hz}, 7-\mathrm{H} \beta$ ); 2.18 (qd, $1 \mathrm{H}, J=11.1,3.2 \mathrm{~Hz}, 8-\mathrm{H}) ; 2.38$ (br dt, $1 \mathrm{H}$, $J=16.8,4.1 \mathrm{~Hz}, 2-\mathrm{H} \alpha) ; 2.47$ (dd, $1 \mathrm{H}, J=19.6,9.4 \mathrm{~Hz}, 16-\mathrm{H} \beta)$; $2.50(\mathrm{dd}, 1 \mathrm{H}, J=17.4,14.8,5.0 \mathrm{~Hz}, 2-\mathrm{H} \beta) ; 4.38(\mathrm{t}, 1 \mathrm{H}, J=2.8 \mathrm{~Hz}$, $6-\mathrm{H} \alpha) ; 5.81(\mathrm{~s}, 1 \mathrm{H}, 4-\mathrm{H})$.

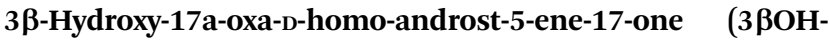
lactone). ${ }^{1} \mathrm{H}$ NMR (600 MHz) (ppm) (CDCl3) $\delta: 0.89$ (s, 3H, 19-H); 1.08-1.16 (m, 2H, 1-H $\alpha, 9-\mathrm{H}) ; 1.32$ (s, 3H, 18-H); 1.32-1.39 (m, $2 \mathrm{H}, 8-\mathrm{H}, 11-\mathrm{H} \alpha$ ); 1.42 (ddd, $1 \mathrm{H}, J=12.5,10.6,4.2 \mathrm{~Hz}, 14-\mathrm{H}$ ); 1.49-1.56 (m, 1H, 2- $\mathrm{H} \alpha, 15-\mathrm{H} \alpha)$; 1.60-1.70 (m, 2H, 7-H $\alpha, 12-\mathrm{H} \alpha)$; $1.79(\mathrm{dq}, 1 \mathrm{H}, J=13.1,3.6 \mathrm{~Hz}, 11-\mathrm{H} \beta) ; 1.83-1.88(\mathrm{~m}, 1 \mathrm{H}, 1-\mathrm{H} \beta, 2-$ $\mathrm{H} \beta$ ); 1.91-1.99 (m, 1H, 12-H $\beta, 15-\mathrm{H} \beta$ ); 2.14-2.20 (m, 1H, 7-H $\beta$ ); $2.23(\mathrm{dq}, 1 \mathrm{H}, J=13.2,3.1 \mathrm{~Hz}, 4-\mathrm{Ha}) ; 2.33(\mathrm{ddd}, 1 \mathrm{H}, J=13.2,5.1$, $2.2 \mathrm{~Hz}, 4-\mathrm{H} \beta$ ); 2.57 (d, $1 \mathrm{H}, J=19.0,9.1 \mathrm{~Hz}, 16-\mathrm{H} \alpha$ ); 2.69 (ddd, $1 \mathrm{H}, J=19.0,8.7,2.2 \mathrm{~Hz}, 16-\mathrm{H} \beta) ; 3.53(\mathrm{tt}, 1 \mathrm{H}, J=11.5,4.6 \mathrm{~Hz}, 3-$ $\mathrm{H} \alpha) ; 5.35(\mathrm{dt}, 1 \mathrm{H}, J=7.1,1.8 \mathrm{~Hz}, 6-\mathrm{H})$.

17a-oxa-D-homo-androst-4-ene-17-one (testololactone). ${ }^{1} \mathrm{H}$ NMR (600 MHz) (ppm) $\left(\mathrm{CDCl}_{3}\right) \delta: 1.03-1.15(\mathrm{~m}, 2 \mathrm{H}, 7-\mathrm{H} \alpha, 9-\mathrm{H})$; 1.14 (s, 3H, 18-H); 1.26-1.32 (m, 1H, 11-H $\beta) ; 1.32$ (s, 3H, 19-H); 1.36-1.43 (m, 2H, 8-H, 14-H); 1.48-1.57 (m, 1H, 15-H $\beta) ; 1.64$ (td, $1 \mathrm{H}, J=13.3,4.2 \mathrm{~Hz}, 12-\mathrm{H} \alpha) ; 1.70(\mathrm{td}, 1 \mathrm{H}, J=13.9,5.0 \mathrm{~Hz}, 1-\mathrm{H} \alpha)$; 1.77 (dq, $J=13.6,3.7 \mathrm{~Hz}, 1 \mathrm{H}, 11-\mathrm{H} \alpha)$; 1.96-2.05 (m, 4H, 1-H $\beta$, 7$\mathrm{H} \beta, 12-\mathrm{H} \beta, 15-\mathrm{H} \alpha) ; 2.27-2.36$ (m, 3H, 2-H $\alpha, 6-\mathrm{H} \alpha, 6-\mathrm{H} \beta) ; 2.40$ (ddd, $1 \mathrm{H}, J=17.0,14.4,5.1 \mathrm{~Hz}, 2-\mathrm{H} \beta) ; 2.56$ (dt, $1 \mathrm{H}, J=18.8$, $9.1 \mathrm{~Hz}, 16-\mathrm{H} \alpha$ ); 2.67 (ddd, $1 \mathrm{H}, J=19.0,8.8,2.2 \mathrm{~Hz}, 16-\mathrm{H} \beta$ ); 5.72 (br s, $1 \mathrm{H}, 4-\mathrm{H})$.

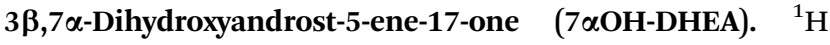
NMR (600 MHz) (ppm) $\left(\mathrm{CDCl}_{3}\right) \delta: 0.87(\mathrm{~s}, 3 \mathrm{H}, 18-\mathrm{H}) ; 1.01(\mathrm{~s}, 3 \mathrm{H}$, $19-\mathrm{H}) ; 1.11(\mathrm{td}, 1 \mathrm{H}, J=13.4,3.8 \mathrm{~Hz}, 1-\mathrm{H} \alpha)$; 1.23-1.31 (m, 2H, 9$\mathrm{H}, 12-\mathrm{H} \alpha) ; 1.49(\mathrm{td}, 1 \mathrm{H}, J=13.1,4.3 \mathrm{~Hz}, 11-\mathrm{H} \alpha) ; 1.50-1.60(\mathrm{~m}$, $2 \mathrm{H}, 2-\mathrm{H} \alpha, 15-\mathrm{H} \alpha) ; 1.64-1.72(\mathrm{~m}, 2 \mathrm{H}, 8-\mathrm{H}, 11-\mathrm{H} \beta) ; 1.78(\mathrm{td}, 1 \mathrm{H}, J$ $=12.1,5.3 \mathrm{~Hz}, 14-\mathrm{H})$; 1.80-1.89 (m, 3H, $1-\mathrm{H} \beta, 2-\mathrm{H} \beta, 12-\mathrm{H} \beta)$; 2.07-2.17 (m, 2H, 15-H $\beta, 16-\mathrm{H} \alpha$ ); 2.29 (br t, $1 \mathrm{H}, J=12.3 \mathrm{~Hz}$, 4$\mathrm{H} \alpha$ ); 2.35 (ddd, $1 \mathrm{H}, J=13.3,4.8,2.0 \mathrm{~Hz}, 4-\mathrm{H} \beta) ; 2.33(\mathrm{dd}, 1 \mathrm{H}, J=$
13.1, $4.6 \mathrm{~Hz}, 16-\mathrm{H} \beta) ; 3.56(\mathrm{tt}, 1 \mathrm{H}, J=11.3,4.7 \mathrm{~Hz}, 3-\mathrm{H} \alpha) ; 3.96(\mathrm{br}$ $\mathrm{t}, 1 \mathrm{H}, J=3.8 \mathrm{~Hz}, 7-\mathrm{H} \beta$ ); 5.63 (dd, $1 \mathrm{H}, J=5.1,1.2 \mathrm{~Hz}, 6-\mathrm{H})$.

Androst-5-ene-3 $\beta, 7 \alpha, 17 \alpha$-triol $\quad\left(3 \beta, 7 \alpha, 17 \alpha\right.$-triol). ${ }^{1} \mathrm{H} \quad \mathrm{NMR}$ $(600 \mathrm{MHz})(\mathrm{ppm})\left(\mathrm{CDCl}_{3}\right) \delta: 0.69(\mathrm{~s}, 3 \mathrm{H}, 18-\mathrm{H}) ; 1.00(\mathrm{~s}, 3 \mathrm{H}, 19-\mathrm{H})$; $1.14(\mathrm{td}, 1 \mathrm{H}, J=13.5,3.7 \mathrm{~Hz}, 1-\mathrm{H} \alpha) ; 1.20-1.29(\mathrm{~m}, 2 \mathrm{H}, 9-\mathrm{H}, 12-$ $\mathrm{H} \alpha)$; 1.44-1.70 (m, 6H, 2-H $\alpha, 8-\mathrm{H}, 11-\mathrm{H} \alpha, 11-\mathrm{H} \beta, 15-\mathrm{H} \alpha, 16-\mathrm{H} \alpha)$; 1.78-1.93 (m, 5H, 1-H $\beta, 2-\mathrm{H} \beta, 12-\mathrm{H} \beta, 14-\mathrm{H}, 15-\mathrm{H} \beta)$; 2.21 (m, 1H, $16-\mathrm{H} \beta$ ); 2.29 (br, $J=13.1 \mathrm{~Hz}, 4-\mathrm{Ha}) ; 2.36$ (ddd, $1 \mathrm{H}, J=13.1,5.0$, $2.1 \mathrm{~Hz}, 4-\mathrm{H} \beta) ; 3.59$ (tt, $1 \mathrm{H}, J=11.0,4.7 \mathrm{~Hz}, 3-\mathrm{H} \alpha) ; 3.77$ (d, $1 \mathrm{H}, J=$ $6.0 \mathrm{~Hz}, 17-\mathrm{H} \beta) ; 3.88$ (br t, $1 \mathrm{H}, J=3.7 \mathrm{~Hz}, 7-\mathrm{H} \beta) ; 5.62(\mathrm{dd}, 1 \mathrm{H}, J=$ $5.3,1.5 \mathrm{~Hz}, 6-\mathrm{H})$.

3 $\beta$-Hydroxyandrost-5-ene-7,17-dione (7Oxo-DHEA). ${ }^{1} \mathrm{H}$ NMR $(600 \mathrm{MHz})(\mathrm{ppm})\left(\mathrm{CDCl}_{3}\right) \delta: 0.89(\mathrm{~s}, 3 \mathrm{H}, 18-\mathrm{H}) ; 1.22$ (s, 3H, 19-H); 1.23-1.34 (m, 2H, 1-H $\alpha, 12-\mathrm{H} \alpha$ ); 1.54-1.68 (m, 4H, 2-H $\alpha, 9-\mathrm{H}, 11-$ $\mathrm{H} \alpha, 14-\mathrm{H}) ; 1.70-1.91(\mathrm{~m}, 3 \mathrm{H}, 11-\mathrm{H} \beta, 12-\mathrm{H} \beta, 15-\mathrm{H} \alpha) ; 1.91-2.00$ $(\mathrm{m}, 2 \mathrm{H}, 1-\mathrm{H} \beta, 2-\mathrm{H} \beta) ; 2.14$ (dd, $1 \mathrm{H}, J=18.8,9.8 \mathrm{~Hz}, 16-\mathrm{H} \alpha)$; 2.352.45 (m, 2H, 4-Ha, 8-H); 2.46 (dd, $1 \mathrm{H}, J=18.8,8.3 \mathrm{~Hz}, 16-\mathrm{H} \beta$ ); 2.54 (ddd, $1 \mathrm{H}, J=13.9,4.6,2.2 \mathrm{~Hz}, 4-\mathrm{H} \beta$ ); 2.81 (ddd, $1 \mathrm{H}, J=$ 13.4, 8.7, 7.2 Hz, 15-H $\beta$ ); 3.68 (tt, $1 \mathrm{H}, J=11.3,4.8 \mathrm{~Hz}, 3-\mathrm{H} \alpha) ; 5.74$ (br s, 1H, 6-H).

\section{Discussion of results}

In order to find new biocatalysts for effective transformations of DHEA, samples of soil and air were collected from the area of Wrocław (Poland). Using the method of serial dilutions, 25 pure strains were isolated. All the strains were tested with respect to their ability to transform DHEA. The most effective strains were identified using molecular techniques. The strains selected for biotransformation on a large scale transformed the substrate with high conversion efficiency (Table 4). All the products were characterized based on their spectral data: ${ }^{1} \mathrm{H}$ and ${ }^{13} \mathrm{C}$ NMR (Table 2), HMBC and HMQC correlation spectra and analysis by GC and TLC.

Selected five fungal strains that were used in this study. All strains were genetically identified by determination of the ITS1ITS2 rDNA sequence (Table 3). The DNA fragments were amplified using PCR with ITS4-ITS5 primers (Table 1), and sequenced. The complete sequences of these products were compared with reference ITS sequences deposited in the GenBank Database (Table 3).

Aspergillus versicolor KCh TJ1. As a result of biotransformation of DHEA by the strain Aspergillus versicolor KCh TJ1, oxidation of the hydroxyl group at C-3 along with isomerisation of the double bond into $\mathrm{C}-4$ position, lead to formation of $\mathrm{AD}$ (Scheme 1). Then two competitive reactions were observed: 1,2dehydration and hydroxylation at C-6. The majority of formed $\mathrm{AD}$ is transformed to $\mathrm{ADD}$, whereas both $\mathrm{AD}$ and $\mathrm{ADD}$ are consequently transformed to corresponding hydroxyderivatives: $6 \beta$-hydroxyandrost-4-ene-3,17-dione $\quad(6 \beta \mathrm{OH}-\mathrm{AD})$ and $6 \beta$ hydroxyandrost-1,4-diene-3,17-dione (6ßOH-ADD).

Introduction of a double bond between $\mathrm{C} 1$ and $\mathrm{C} 2$ of a steroid may increase biological activity of the newly formed compound. Chemical methods of obtaining 1-ene-steroids are usually multi-step processes and may lead to spontaneous aromatisation of the A ring. In contrast, biological methods are efficient and selective. ${ }^{27}$ The majority of literature reports on 
microbial dehydrogenation in $\mathrm{C} 1-\mathrm{C} 2$ positions relate to bacteria of the genera Mycobacterium, Rhodococcus, Nocardia, and Arthrobacter. ${ }^{28,29}$ Unfortunately, introduction of a double bond to the A ring by bacteria is the beginning of the steroid degradation pathway. ${ }^{30}$ There are many reports of using bacterial 3-ketosteroid $\Delta^{1}$-dehydrogenase enzymes fom Arthrobacter simplex or Gordonia neofelifaecis NRRL B-59395 in transformation of various 3-on-4-en steroids, although conversion of $3 \beta$-ol-4-en was unsuccessful. ${ }^{27,31-34}$ It has been found that steroid compounds (cholesterol, progesterone, pregnenolone, $\mathrm{AD}$, DHEA and testosterone) were transformed to ADD by the Fusarium fungi, however, with lower yields since ADD was an

Table 3 Identification of fungal strains on the basis of the sequence of the ITS1-ITS2 sequences and comparison with reference ITS sequences

Name of

fungal Identified fungal

strain species Sequence identity

KCh S1 Fusarium acuminatum

99\% identity to the Fusarium acuminatum, acc. numbers: KT965739.1, KR051403.1, KR051401.1

KCh W2 Mucor hiemalis

KCh S4 Penicillium chrysogenum

KCh W7 Penicillium commune

$100 \%$ identity to the Penicillium

\section{KCh TJ1 Aspergillus} versicolor
99\% identity to the Penicillium chrysogenum acc. numbers: KY218708.1, KY218694.1, KY218692.1 commune, acc. numbers: KP120886.2, JN368450.1, HQ652873.1

$100 \%$ identity to the Mucor hiemalis acc. numbers: KT583167.1, KP942918.1, KM280062.1

$$
\text { HQ652873.1 }
$$

99\% identity to the Aspergillus versicolor acc. numbers: KF691800.1, LC105698.1, LC105694.1
Sequence

TCTACTGATCCGAGGTCACATTCAGAAGTTGGGGTTTTACGGCATGGCC GCGCCGCGTTCCAGTTGCGAGGTGTTAGCTACTACGCAATGGAGGCTG CAGCGAGACCGCCAATGTATTTCGGGGGCGGCACCGCCCAGAAGGGC AGAGCCGATCCCCAACACCAAACCCGGGGGCTTGAGGGTTGAAATGAC GCTCGAACAGGGCATGCCCGCCGGAATACCAGCGGGCGCAATGTGCGT TCAAAGATTCGATGATTCACTGAATTCTGCAATTCACATTACTTATCGCA TTTTGCTGCGTTCTTCATCGATGCCAGAACCAAGAGATCCGTTGTTGAAA GTTTTGATTTATTTGTTTGTTTTACTCAGAAGTTACAATAAGAAACATTAG AGTTTGGGTCCTCTGGCGGGCCGTCCCGTTTTACGGGGCGCGGGCTGA TCCGCCGAGGCAACATTAAGGTATGTTCACAGGGGTTTGGGAGTTGTAA ACTCGGTAATGATCCCTCCGCTGGTTCACCAAC

ATATGCTTAAGTTCAGCGGGTAATCCCGCCTGATTTCAGATCAAATTTAA GAATGATTTATTTGGGAGGCCCCAGAGATAGTCTTTTTACTAGAGCATTC CTTTATATTAAAAAAATGTTCAGGCAGAAAGAACAATAGTTCAGGCCTAAT AATTTTAAAGAATTCAGCAATAAAGCCGAAACTCTCATTTCCATTCACAAC AAAATTATGAATGTGGGGTGTTTTTGATACTGAAACAGGCGTGCTCAATG GAATACCATTGAGCGCAAGTTGCGTTCAAAGACTCGATGATTCACTGAAT ATGCAATTCACACTAGTTATCGCACTTTGCTACGTTCTTCATCGATGCGA GAACCAAGAGATCCGTTGTTAAAAGTTGTTTTATAAGTTTTTTACGCTTA TGTTACAATTATAATACTGAATTCTTTTGGTAAATAATTAATAGGATACCA GGCCTAAGCCTGACTTTCAGCTCGGTTAACATTCTAGTAGCCTATCCCTA TGGCTAAAAGACATTCCTCAGACGCTGAAAAATTAAAACAGTTCACAGTA AAAAAGAATGAACCATTCGCTAGAAAACTAGCAAAGGCCATCTAAATTAT TTAT

TGCGGAGGACATTACCGAGTGAGGGCCCTCTGGGTCCAACCTCCCACC CGTGTTTATTTTACCTTGTTGCTTCGGCGGGCCCGCCTTAACTGGCCGC CGGGGGGCTTACGCCCCCGCTGCCTTTCGGGCCCGTCCCCCGGGATCG GAGGACGGGGCCCAACACACAAGCCGTGCTTGAGGGCAGAAATGACGC TCGGACAGGCATGCCCCCCGGAATACCAGGgGGCGCAATGTGCGTTCAA AGACTCGATGATT

CTGATCGAGGTCACCTGGATAAAAATTTGGGTTGATCGGCAAGCGCCGG CCGGGCCTACAGAGCGGGTGACAAAGCCCCATACGCTCGAGGACCGGA CGCGGTGCCGCCGCTGCCTTTCGGGCCCGTCCCCCGGGATCGGAGGA CGGGGCCCAACACACAAGCCGTGCTTGAGGGCAGCAATGACGCTCGGA CAGGCATGCCCCCCGGAATACCAGGGGGCGCAATGTGCGTTCAAAGAC TCGATGATTCACTGAATTTGCAATTCACATTACGTATCGCATTTCGCTGC GTTCTTCATCGATGCCGGAACCAAGAGATCCGTTGTTGAAAGTTTTAAAT AATTTATATTTTCACTCAGACTACAATCTTCAGACAGAGTTCGAGGGTGT CTTCGGCGGGCGCGGGCCCGGGGGCGTAAGCCCCCCGGCGGCCAGTT AAGGCGGGCCCGCCGAAGCAACAAGGTAAAATAAACACGGGTGGGAGG TTGGACCCAGAGG

CGGAGGACATTACTGAGTGCGGGCTGCCTCCGGGCGCCCAACCTCCCA CCCGTGAATACCTAACACTGTTGCTTCGGCGGGGAACCCCCTCGGGGG CGAGCCGCCGGGGACTACTGAACTTCATGCCTGAGAGTGATGCAGTCTG AGTCTGAATATAAAATCAGTCAAAACTTTCAACAATGGATCTCTTGGTTC CGGCATCGATGAAGAACGCAGCGAACTGCGATAAGTAATGTGAATTGCA GAATTCAGTGAATCATCGAGTCTTTGAACGCACATTGCGCCCCCTGGCA TTCCGGGGGGCATGCCTGTCCGAGCGTCATTGCTGCCCATCAAGCCCG GCTTGTGTGTTGGGTCGTCGTCCCCCCCGGGGGACGGGCCCGAAAGG CAGCGGCGGCACCGTGTCCGGTCCTCGAGCGTATGGGGCTTTGTCACC CGCTCGACTAGGGCCGGCCGGGCGCCAGCCGACGTCTCCAACCATTTT TCTTCAGGTTGA 
Table 4 Products accumulation during the conversion of DHEA

\begin{tabular}{|c|c|c|c|c|c|c|}
\hline \multirow[b]{2}{*}{ Strain } & \multirow{2}{*}{$\begin{array}{l}\text { Compounds found } \\
\text { in the reaction mixture }\end{array}$} & \multirow{2}{*}{$\begin{array}{l}\text { Retention time } \\
\text { by GC }[\mathrm{min}]\end{array}$} & \multirow{2}{*}{$\begin{array}{l}\text { Retention factor } \\
\text { by TLC }\end{array}$} & \multicolumn{3}{|c|}{$\begin{array}{l}\text { Biotransformation time }^{a} \\
\text { [days] }\end{array}$} \\
\hline & & & & 1 & 3 & 7 \\
\hline \multirow[t]{3}{*}{ Aspergillus versicolor $\mathrm{KCh}$ TJ1 } & DHEA & 4.9 & 0.62 & 89 & - & - \\
\hline & $\mathrm{AD}$ & 6.0 & 0.65 & 5 & 88 & 6 \\
\hline & $\mathrm{ADD}$ & 6.4 & 0.60 & - & 2 & 56 \\
\hline \multirow[t]{4}{*}{ Penicillium commune $\mathrm{KCh}$ W7 } & DHEA & 4.9 & 0.62 & 65 & 0 & 0 \\
\hline & $\mathrm{AD}$ & 6.0 & 0.65 & 22 & 0 & 0 \\
\hline & $3 \beta \mathrm{OH}$-lactone & 8.6 & 0.48 & 3 & 11 & 9 \\
\hline & Testololactone & 10.2 & 0.50 & 1 & 85 & 77 \\
\hline \multirow[t]{2}{*}{ Penicillium chrysogenum KCh S4 } & DHEA & 4.9 & 0.62 & 26 & 0 & - \\
\hline & $\mathrm{AD}$ & 6.0 & 0.65 & 61 & 22 & - \\
\hline & $7 \alpha \mathrm{OH}-\mathrm{DHEA}$ & 6.9 & 0.35 & 12 & 34 & 43 \\
\hline & 7Oxо-DHEA & 7.4 & 0.54 & 0 & 2 & 3 \\
\hline \multirow[t]{2}{*}{ Fusarium acuminatum $\mathrm{KCh} \mathrm{S} 1$} & DHEA & 4.9 & 0.62 & 76 & 12 & 0 \\
\hline & $7 \alpha \mathrm{OH}-\mathrm{DHEA}$ & 6.9 & 0.35 & 21 & 85 & 97 \\
\hline
\end{tabular}

intermediate product. ${ }^{16,35}$ Also Trichoderma hamatum strain has ability to transform different 3-one-4-en steroid substrates to 1,4-diene derivatives. ${ }^{36}$ Obtained with high yield ADD may be a key intermediate in synthesis of many sex hormones, aromatase inhibitors and also immunomodulatory drugs..$^{37-39}$

Penicillium commune KCh W7. In the culture of the Penicillium commune KCh W7 DHEA was at first isomerized and oxidized to $\mathrm{AD}$, and subsequently underwent Baeyer-Villiger oxidation to testololactone (Scheme 2). In a small amount (about $10 \%$ ) the product of $\mathrm{D}$ ring lactonization, 17a-oxa-Dhomo-androst-5-ene-17-one (3ßOH-lactone), was observed. 17aoxa-D-homo-androst-5-ene-17-one is the precursor of testolactone, which is used as aromatase inhibitor in treatment of hormone-dependent cancers.
High ability to perform Baeyer-Villiger oxidation in a D ring of steroids was observed for filamentous fungi of the genera Aspergillus and Penicillium and, in smaller degree, for Beauveria, Chaetomium, Fusarium, Mucor, and Rhizopus. ${ }^{40}$ Conversions of cyclic ketones to corresponding lactones are catalysed by Baeyer-Villiger monooxygenases (BVMO) (E.C. 1.14.13.x). ${ }^{41}$ These class of enzymes introduce a single atom of molecular oxygen into the substrate while the other one is reduced to water. They use a FAD or FNM as a prosthetic group and require NADPH or NADH as a cofactor. ${ }^{41}$

An analysis of the available literature reports revealed three possible transformations pathways:

1. DHEA may be transformed directly to $3 \beta \mathrm{OH}-$ lactone: Penicillium lilacinum AM111, Aspergillus tamarii KITA; ${ }^{42,43}$<smiles>CC12CCC3C(CC=C4CC(O)CCC43C)C1CCC2=O</smiles><smiles>CC1(C)C(=O)CCC2(C)C3CCC4=CC(=O)CCC4(C)C3CCC12</smiles>
DHEA

AD

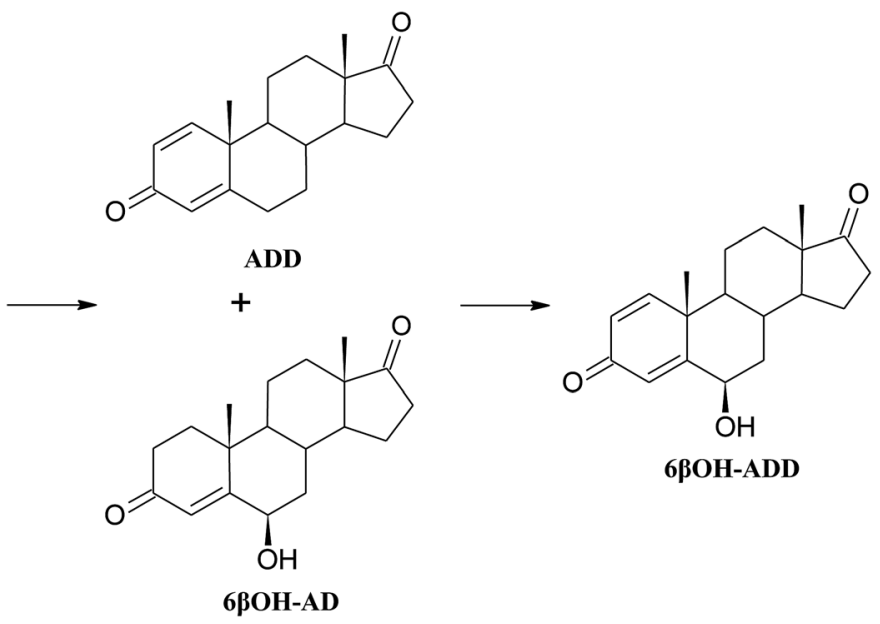

Scheme 1 Metabolism of DHEA by Aspergillus versicolor KCh TJ1. 
<smiles>CC1CCC2C3CC=C4CC(O)CCC4(C)C3CCC12C</smiles><smiles>CC12CCC3C(CC=C4CC(O)CCC43C)C1CCC(=O)O2</smiles>

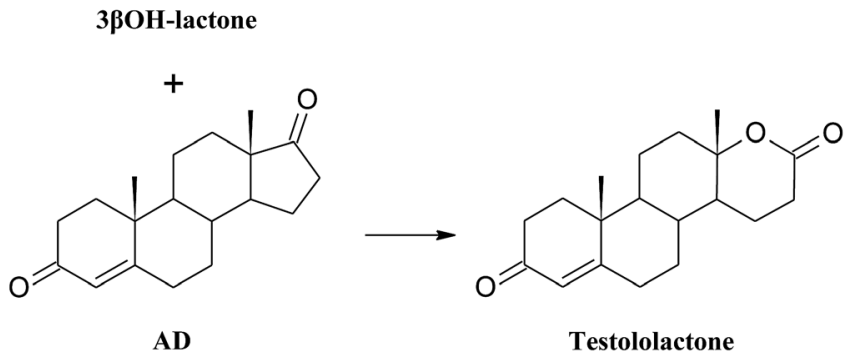

Scheme 2 Baeyer-Villiger oxidation of DHEA by Penicillium commune KCh W7.

2. At first isomerization and oxidation of 3-hydroxy-5-ene moiety to 3-oxo-4-ene occure, and then BV oxidation of the D ring takes place - Penicillium griseopurpureum, Penicillium lanosocoeruleum; ${ }^{44,45}$

3. At first BV oxidation to $3 \beta \mathrm{OH}$-lactone occurs and then oxidation of the hydroxyl group at C-3 takes place, leading to formation of testololactone: Penicillium simplicissimum WY1342, Aspergillus parasitic. ${ }^{46,47}$

According to the literature, it is possible that the two reactions occur simultaneously, as in the case of Penicillium glabrum and Penicillium camemberti AM83. ${ }^{44,48}$ In the culture of Penicillium commune $\mathrm{KCh} \mathrm{W} 7$ strain lactonization was observed both in the substrate (5-ene-3-ol moiety), and in the formed in biotransformation AD (5-ene-3-one moiety). Moreover, $3 \mathrm{BOH}-$ lactone was not transformed to testololactone. Literature review indicates that isolated Baeyer-Villiger monooxygenases (BVMO) can transform DHEA to 3 $\beta$-hydroxy-17a-oxa-D-homoandrost-5-ene-17-one in higher yield than $\mathrm{AD}$ to 17a-oxa-Dhomo-androst-4-ene-17-one. ${ }^{49}$ Additionally in the culture of Beauveria bassiana, Penicillium camemberti AM83 and P. lilacinum AM111 AD was transformed slower than DHEA into corresponding lactones. ${ }^{42,48,50}$

Penicillium chrysogenum KCh S4. Transformations of DHEA in the culture of this strain proceeded according to the previously proposed pathway no. 2 (Scheme 3). Similarly to transformations described earlier, we observed the degradation of formed testololactone was observed. The same process of metabolizing DHEA was observed for transformation in the Penicillium citreo-viride culture. ${ }^{51}$
Mucor hiemalis KCh W2. In culture of the Mucor hiemalis KCh W2 strain, transformation of the substrate proceeded in two directions: substrate underwent a stereoselective hydroxylation in $7 \alpha$ position, followed by oxidation of the hydroxyl group to 7oxoDHEA (Scheme 4). Reduction of the carbonyl group at C-17, followed by hydroxylation at $7 \alpha$ position formed androst-5-ene$3 \beta, 7 \alpha, 17 \alpha$-triol, which is a major transformation. Conversion of inactive 17 oxo form to active $17 \beta$-hydroxy-form and vice versa is catalysed by $17 \beta$-hydroxysteroid dehydrogenase (17 $\beta$-HSD). ${ }^{52}$ This is a large group of enzymes different in structure, tissue distribution, substrate specificity and mechanism of regulation. ${ }^{53}$ Due to their high selectivity they can be used in the efficient testosterone production..$^{54}$

$3 \beta, 7 \alpha, 17 \alpha$-Trihydroxyandrost-5-ene shows high anticancer activity toward lymphoma. ${ }^{55}$ It has been proved that androstentriols with such location of the hydroxyl groups have the most potent anticancer activity. A chemical method of obtaining $3 \beta, 7 \alpha, 17 \alpha$-trihydroxyandrost-5-ene from $3 \beta, 17 \alpha-$ dihydroxyandrost-5-ene by selective oxidation of allylic carbon atom and reduction of the newly formed carbonyl group is known. ${ }^{55}$ However, to the best of our knowledge, there is no literature report on microbial method of obtaining $3 \beta, 7 \alpha, 17 \alpha-$ trihydroxyandrost-5-ene.

Fusarium acuminatum KCh S1. In the culture of the Fusarium acuminatum KCh S1 strain we observed stereospecific hydroxylation of DHEA at $7 \alpha$ position, leading to the single product: $7 \propto \mathrm{OH}-\mathrm{DHEA}$ with $100 \%$ yield (Scheme 5) was observed. Prolongation, longer than 6 days, results in product degradation.

An example of identical transformation of DHEA and some other 5 -ene steroids to $7 \alpha$-hydroxyderivatives by the strain

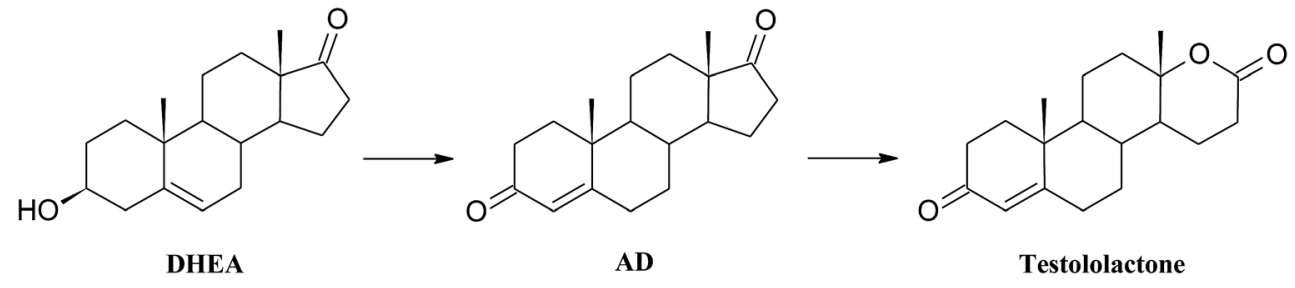

Scheme 3 Lactonization of DHEA in the culture of Penicillium chrysogenum KCh S4. 
<smiles>CC1CCC2C3CC=C4CC(O)CCC4(C)C3CCC12C</smiles><smiles>CC1CCC2C(C1)C(=O)C=C1CC(O)CCC12C</smiles><smiles></smiles>

Scheme 4 Products of transformation of DHEA in the Mucor hiemalis KCh W2 culture.<smiles>CC1CCC(=O)C2(C)CCC3C4CC=C5CC(O)CCC5(C)C4CCC3C12</smiles>

Scheme 5 Hydroxylation of DHEA by Fusarium acuminatum KCh S1.

Fusarium culmorum has been reported. ${ }^{56}$ Similar reactions of selective hydroxylation were also performed by Fusarium moniliforme verticillioides, F. oxysporum, Gelasinospora retispora and Mucor silvaticus, ${ }^{57-60}$ as well as by non-selective ones: Absidia coerulea AM93 ${ }^{61}$ and Mortierella isabellina AM212. ${ }^{62}$ 7-Hydroxyderivatives of DHEA are interesting due to their neuroprotective ${ }^{63}$ and immunostimulatory properties ${ }^{64}$ and also antiinflammatory activity without the glucocorticoid action., ${ }^{5,19}$ 7Hydroxyderivatives of DHEA may also be regarded as epiandrosterone derivative with proven neuroprotective activity both in vitro and in vivo. ${ }^{65}$

\section{Conclusions}

Among 25 fungal strains isolated from the area of Wrocław, we selected 5 strains which were the most efficient in transformation of dehydroepiandrosterone and we used them for the biotransformation on larger scale, in order to identify the metabolites. Additionally, the strains were subjected to genetic identification.

All the products were purified by chromatographic methods. Based on GC, TLC analysis and ${ }^{1} \mathrm{H}$ NMR, ${ }^{13} \mathrm{C}$ NMR spectra (including correlation spectra) we established the structures of nine products.

Three compounds were found to be new metabolites of DHEA. To the best of our knowledge, there are no reports in literature on transformations of DHEA leading to: adrost-1,4diene-3,17-dione, 6 $\beta$-hydroxyandrost-1,4-diene-3,17-dione and androst-5-ene-3 $3,7 \alpha, 17 \alpha$-triol.
The majority of tested biocatalysts showed excellent selectivity in the transformation of DHEA. Due to this feature, they possibly may be used for industrial production of bioactive DHEA metabolites provided basic genetic engineering techniques successfully increase the content of specific enzymes in these biocatalysts.

\section{Acknowledgements}

Publication supported by the Wroclaw Centre of Biotechnology under the Leading National Research Centre (KNOW) programme for years 2014-2018. Special thanks to Jarosław Popłoński for language correction.

\section{References}

1 M. V. Donova and O. V. Egorova, Appl. Microbiol. Biotechnol., 2012, 94, 1423-1447.

2 M. M. Chen, F. Q. Wang, L. C. Lin, K. Yao and D. Z. Wei, Appl. Microbiol. Biotechnol., 2012, 96, 133-142.

3 R. M. Loria, Steroids, 2002, 67, 953-966.

4 O. Hennebert, M. A. Pelissier, S. Le Mee, E. Wülfert and R. Morfin, J. Steroid Biochem. Mol. Biol., 2008, 110, 255-262.

5 M. A. Pélissier, C. Muller, M. Hill and R. Morfin, Steroids, 2006, 71, 240-248.

6 B. Brazzini and N. Pimpinelli, Am. J. Clin. Dermatol., 2002, 3, 47-58.

7 M. Panjari and S. R. Davis, Hum. Reprod. Update, 2007, 13, 239-248.

8 K. Rutkowski, P. Sowa and J. R.-T. A. Kuryliszyn-Moskal, Drugs, 2014, 74, 1195-1207.

9 F. Hammer, S. Subtil, P. Lux, C. Maser-Gluth, P. M. Stewart, B. Allolio and W. Arlt, J. Clin. Endocrinol. Metab., 2005, 90, 3600-3605.

10 D. Liu and J. S. Dillon, J. Biol. Chem., 2002, 277, 21379-21388.

11 X. Ding, D. Wang, L. Li and H. Ma, Int. J. Biochem. Cell Biol., 2016, 70, 126-139.

12 T. Suzuki, N. Suzuki, R. A. Daynes and E. G. Engleman, Clin. Immunol. Immunopathol., 1991, 61, 202-211. 
13 M. Maggio, F. De Vita, A. Fisichella, E. Colizzi, S. Provenzano, F. Lauretani, M. Luci, G. Ceresini, E. Dall, P. Caffarra, G. Valenti and G. Paolo, J. Steroid Biochem. Mol. Biol., 2015, 145, 281-292.

14 D. T. Villareal, J. O. Holloszy and W. M. Kohrt, Clin. Endocrinol., 2000, 53, 561-568.

15 M. Panjari, R. J. Bell, F. Jane, J. Adams, C. Morrow and S. R. Davis, Maturitas, 2009, 63, 240-245.

16 H. Zhang, J. Ren, Y. Wang, C. Sheng, Q. Wu, A. Diao and D. Zhu, Tetrahedron, 2013, 69, 184-189.

17 P. Fernandes, A. Cruz, B. Angelova, H. M. Pinheiro and J. M. S. Cabral, Enzyme Microb. Technol., 2003, 32, 688-705.

18 R. Hampl, O. Lapčík, M. Hill, J. Klak, A. Kasal, A. Nováček, I. Šterzl, J. Šterzl and L. Stárka, Physiol. Res., 2000, 49.

19 M.-A. Pelissier, C. Trap, M.-I. Malewiak and R. Morfin, Steroids, 2004, 69, 137-144.

20 K. Kristan and T. L. Rižner, J. Steroid Biochem. Mol. Biol., 2012, 129, 79-91.

21 H. Pellissier and M. Santelli, Org. Prep. Proced. Int., 2001, 33, 1-58. 22 T. Janeczko, N. Milecka and E. Kostrzewa-Susłow, Przem. Chem., 2012, 91, 767-771.

23 C. N. Ahlem, T. M. Page, D. L. Auci, M. R. Kennedy, K. Mangano, F. Nicoletti, Y. Ge, Y. Huang, S. K. White, S. Villegas, D. Conrad, A. Wang, C. L. Reading and J. M. Frincke, Steroids, 2011, 76, 145-155.

24 D. L. Auci, C. L. Reading and J. M. Frincke, Autoimmun. Rev., 2009, 8, 369-372.

25 Ł. Stępień, M. Jestoi and J. Chełkowski, World Mycotoxin J., 2013, 6, 399-409.

26 T. J. White, T. Bruns, S. Lee and J. Taylor, in PCR Protocols: a Guide to Methods and Applications, ed. M. A. Innis, D. H. Gelfand, J. J. Sninsky and T. J. White, Academic Press, San Diego, USA, 1990, pp. 315-322.

27 Y. Li, F. Lu, T. Sun and L. Du, Lett. Appl. Microbiol., 2007, 44, 563-568.

28 L. Fernández De Las Heras, R. Van Der Geize, O. Drzyzga, J. Perera and J. María Navarro Llorens, J. Steroid Biochem. Mol. Biol., 2012, 132, 271-281.

29 M. Kisiela, A. Skarka, B. Ebert and E. Maser, J. Steroid Biochem. Mol. Biol., 2012, 129, 31-46.

30 J. K. Capyk, I. Casabon, R. Gruninger, N. C. Strynadka and L. D. Eltis, J. Biol. Chem., 2011, 286, 40717-40724.

31 K. P. Choi, I. Molnár, M. Yamashita and Y. Murooka, J. Biochem., 1995, 117, 1043-1049.

32 Y. Li, F. Lu, T. Sun and L. Du, Lett. Appl. Microbiol., 2007, 44, 563-568.

33 W. Wei, S. Y. Fan, F. Q. Wang and D. Z. Wei, World J. Microbiol. Biotechnol., 2014, 30, 1947-1954.

34 Q. Zhang, Y. Ren, J. He, S. Cheng, J. Yuan, F. Ge, W. Li, Y. Zhang and G. Xie, Ann. Microbiol., 2015, 65, 1961-1971.

35 L. A. R. Sallam, A. M. El-Refai and H. A. El-Minofi, Process Biochem., 2005, 40, 203-206.

36 A. Bartmańska and J. Dmochowska-Gładysz, Enzyme Microb. Technol., 2007, 40, 1615-1621.

37 L. R. Lehman and J. D. Stewart, Curr. Org. Chem., 2001, 5, 439-470.

38 I. Ahmad and Shagufta, Eur. J. Med. Chem., 2015, 102, 375386.
39 M. R. Yadav, M. A. Barmade, R. S. Tamboli and P. R. Murumkar, Eur. J. Med. Chem., 2015, 105, 1-38.

40 L. Butinar, M. Mohorčič, V. Deyris, K. Duquesne, G. Iacazio, M. Claeys-Bruno, J. Friedrich and V. Alphand, Phytochemistry, 2015, 117, 144-153.

41 V. Alphand and R. Wohlgemuth, Curr. Org. Chem., 2010, 14, 1928-1965.

42 T. Kołek, A. Szpineter and A. Świzdor, Steroids, 2008, 73, 1441-1445.

43 A. C. Hunter, E. Coyle, F. Morse, C. Dedi, H. T. Dodd and S.-J. Koussoroplis, Biochim. Biophys. Acta, Mol. Cell Biol. Lipids, 2009, 1791, 110-117.

44 L. H. Huang, J. Li, G. Xu, X. H. Zhang, Y. G. Wang, Y. L. Yin and H. M. Liu, Steroids, 2010, 75, 1039-1046.

45 A. Świzdor, Molecules, 2013, 18, 13812-13822.

46 B. Yang, Y. Wang, X. Chen, J. Feng, Q. Wu and D. Zhu, Tetrahedron, 2014, 70, 41-46.

47 M. L. Mascotti, M. A. Palazzolo, F. R. Bisogno and M. KurinaSanz, Steroids, 2016, 109, 44-49.

48 T. Kołek, A. Szpineter and A. Świzdor, Steroids, 2009, 74, 859862.

49 E. Beneventi, G. Ottolina, G. Carrea, W. Panzeri, G. Fronza and P. C. K. Lau, J. Mol. Catal. B: Enzym., 2009, 58, 164-168.

50 A. Świzdor, T. Kołek, A. Panek and A. Białońska, Biochim. Biophys. Acta, Mol. Cell Biol. Lipids, 2011, 1811, 253-262.

51 H.-M. Liu, H. Li, L. Shan and J. Wu, Steroids, 2006, 71, 931-934.

52 G. Moeller and J. Adamski, Mol. Cell. Endocrinol., 2006, 248, 47-55.

53 P. Lukacik, K. L. Kavanagh and U. Oppermann, Mol. Cell. Endocrinol., 2006, 248, 61-71.

54 M. Shao, X. Zhang, Z. Rao, M. Xu, T. Yang, H. Li, Z. Xu and S. Yang, Green Chem., 2016, 18, 1774-1784.

55 M. R. Graf, W. Jia, M. L. Lewbart and R. M. Loria, Chem. Biol. Drug Des., 2009, 74, 625-629.

56 T. Kołek, J. Steroid Biochem. Mol. Biol., 1999, 71, 83-90.

57 A. C. Cotillon and R. Morfin, J. Steroid Biochem. Mol. Biol., 1999, 68, 229-237.

58 P. Durairaj, S. Malla, S. P. Nadarajan, P.-G. Lee, E. Jung, H. H. Park, B.-G. Kim and H. Yun, Microb. Cell Fact., 2015, 14, 45.

59 M. Koshimura, T. Utsukihara, A. Hara, S. Mizobuchi, C. A. Horiuchi and M. Kuniyoshi, J. Mol. Catal. B: Enzym., 2010, 67, 72-77.

60 Y. Wang, D. Sun, Z. Chen, H. Ruan and W. Ge, Biocatal. Biotransform., 2013, 31, 168-174.

61 N. Milecka-Tronina, T. Kołek, A. Świzdor and A. Panek, Bioorg. Med. Chem., 2014, 22, 883-891.

62 T. Kołek, N. Milecka, A. Świzdor, A. Panek and A. Białońska, Org. Biomol. Chem., 2011, 9, 5414-5422.

63 M. Schumacher, S. Weill-Engerer, P. Liere, F. Robert, R. J. M. Franklin, L. M. Garcia-Segura, J. J. Lambert, W. Mayo, R. C. Melcangi, A. Parducz, U. Suter, C. Carelli, E. E. Baulieu and Y. Akwa, Prog. Neurobiol., 2003, 71, 3-29.

64 R. Morfin, P. Lafaye, A. C. Cotillon and F. Nato, Ann. N. Y. Acad. Sci., 2000, 917, 971-982.

65 A. K. Pringle, W. Schmidt, J. K. Deans, E. Wulfert, K. G. Reymann and L. E. Sundstrom, Eur. J. Neurosci., 2003, 18, 117-124. 